\title{
REVERSE ITERATED FUNCTION SYSTEM AND DIMENSION OF DISCRETE FRACTALS
}

\author{
QI-RONG DENG
}

(Received 27 March 2008)

\begin{abstract}
A reverse iterated function system is defined as a family of expansive maps $\left\{T_{1}, T_{2}, \ldots, T_{m}\right\}$ on a uniformly discrete set $M \subset \mathbb{R}^{d}$. An invariant set is defined to be a nonempty set $F \subseteq M$ satisfying $F=\bigcup_{j=1}^{m} T_{j}(F)$. A computation method for the dimension of the invariant set is given and some questions asked by Strichartz are answered.
\end{abstract}

2000 Mathematics subject classification: 28A80.

Keywords and phrases: reverse iterated function system, dimension, discrete invariant set, overlap.

\section{Introduction}

Fractal structure is characterized by the repetition of detail at small scales. Why not large scales as well? Motivated by this, Strichartz [4] explored two ways to carry this out. He considered a set of expansive mappings $\left\{T_{j}\right\}_{j=1}^{m}$ defined on a discrete complete metric space $M \subset \mathbb{R}^{d}$ and called it a reverse iterated function system (RIFS). He defined the notion of an invariant set which is a union of forward orbits of fixed points of the iterated mappings from the RIFS in [4]. A simple example is the integer Cantor set (all positive integers which are expressible in base three using only zeros and twos as digits) discussed by Bedford and Fisher in [1]. For the case $M=\mathbb{Z}$, Strichartz defined the dimension of invariant sets and gave a method to compute the dimension of invariant sets for the nonoverlapping case, as well as asking some basic questions about the case when nonoverlapping occurs.

If we extend the mappings $\left\{T_{j}\right\}_{j=1}^{m}$ to be defined on $\mathbb{R}^{d}$, then $\left\{T_{j}^{-1}\right\}_{j=1}^{m}$ is a contractive iterated function system (IFS). It can be expected that an invariant set of the RIFS $\left\{T_{j}\right\}_{j=1}^{m}$ is related to the attractor of the IFS $\left\{T_{j}^{-1}\right\}_{j=1}^{m}$. By this observation, we obtain a method to compute the dimension of the invariant set of the RIFS $\left\{T_{j}\right\}_{j=1}^{m}$ for overlapping cases, and three questions asked by Strichartz [4] are also answered.

We first introduce some definitions given in [4]; a small modification is made.

(C) 2009 Australian Mathematical Society 0004-9727/2009 \$16.00 
Definition 1.1. Let $M \subseteq \mathbb{R}^{d}$ be a uniformly discrete set and $\left\{T_{j}\right\}_{j=1}^{m}$ be a family of expansive (under some metric of $\mathbb{R}^{d}$ ) transforms defined on $\mathbb{R}^{d}$. Assume $T_{j}(M) \subseteq M$ for all $j$ :

(i) $\left\{T_{j}\right\}_{j=1}^{m}$ is called an RIFS;

(ii) $P=\left\{x \in M \mid T_{J}(x)=x\right.$ for some $J$ with positive length $\}$;

(iii) a nonempty compact set $F \subseteq M$ is called an invariant set if

$$
F=\bigcup_{j=1}^{m} T_{j}(F)
$$

(iv) if the union in (iii) is disjoint, $F$ is said to be nonoverlapping;

(v) for any $x \in M, F_{x}=\left\{T_{J}(x) \mid J=j_{1} \ldots j_{n}\right.$ with $1 \leq j_{i} \leq m$ and $\left.n>0\right\}$ is called a forward orbit of $x$ for $\left\{T_{j}\right\}_{j=1}^{m}$.

For any invariant set $F$, a basic aspect of $F$ is how dense it is in $M$. Strichartz [4] gave the following definition for the case $d=1, M=\mathbb{Z}$.

DEFINITION 1.2. For an RIFS $\left\{T_{j}\right\}_{j=1}^{m}$ on a uniformly discrete set $M \subseteq \mathbb{R}^{d}$, let $F$ be an invariant set. We define its dimension as

$$
\operatorname{dim} F=\lim _{r \rightarrow+\infty} \log \left(\#\left\{F \cap B_{r}\right\}\right) / \log r
$$

if the limit exists, where $B_{r}$ is the ball centered at the origin with radius $r>0$.

For the case that $\left\{T_{j}(x)=R x+b_{j}\right\}_{j=1}^{m}$ and $F$ is nonoverlapping, Strichartz [4] obtained a method to compute the dimension of $F$, that is, $\operatorname{dim} F=-\ln m / \ln \gamma$.

In general, however, invariant sets are overlapping. Natural questions are: how can we deal with the overlapping cases and when does overlapping occur? Our goal in this paper is to consider these questions. We prove the following result.

THEOREM 1.3. Let $a \in P$; if the RIFS $\left\{T_{j}(x)=R^{k_{j}} x+b_{j}\right\}_{j=1}^{m}$ is a family of similitudes on $\mathbb{R}^{d}$ and the IFS $\left\{S_{j}(x)=R^{-k_{j}}\left(x-b_{j}\right)\right\}_{j=1}^{m}$ satisfies the finite type condition (FTC), then

$$
\operatorname{dim} F_{a}=\operatorname{dim}_{\mathrm{H}}(K),
$$

where $K$ is the attractor of the IFS $\left\{S_{j}\right\}_{j=1}^{m}$.

Ngai and Wang [2] have given a method for the computation of $\operatorname{dim}_{\mathrm{H}}(K)$, so our theorem means that we have been able to compute the dimension of any invariant set of an RIFS satisfying the conditions stated in our theorem.

We arrange the paper as follows. In Section 2, we introduce some results of Strichartz [4]. Then we discuss the questions asked by Strichartz by connecting the related IFS. Section 3 is devoted to considering the computation of the dimensions of invariant sets of the RIFS $\left\{T_{j}\right\}_{j=1}^{m}$ for overlapping cases. Theorem 1.3 is proved there. 


\section{Nonoverlapping and the open set condition}

The next two lemmas are easy to see.

LEMMA 2.1. For any $x \in M, F_{x}$ is a finite set if and only if $F_{x}=\{x\}$, and so $T_{j}(x)=x$ for all $j$.

LEMMA 2.2 [4]. The system $\left\{T_{j}\right\}_{j=1}^{m}$ is an RIFS defined on a uniformly discrete set $M \subset \mathbb{R}^{d}$.

(i) There exists an invariant set $F \subseteq M$ if and only if $P \neq \emptyset$.

(ii) The set $P$ is finite.

(iii) Any invariant set $F$ is a union of forward orbits of points in $P$.

In the following, we consider a self-similar RIFS defined on some uniformly discrete set $M \subset \mathbb{R}^{d}$ :

$$
T_{j}(x)=R_{j} x+b_{j}, \quad j=1,2, \ldots, m,
$$

where $\left\{R_{j}\right\}_{j=1}^{m}$ are similar matrices with expansive ratios $\left\{\gamma_{j}>1\right\}_{j=1}^{m}$, and a self-affine (or self-similar) RIFS:

$$
T_{j}(x)=R x+b_{j}, \quad j=1,2, \ldots, m,
$$

where $R$ is an expanding matrix (which may be an affine or a similar matrix), $b_{j} \in \mathbb{R}^{d}$.

DEFINITION 2.3. The following two iterated function systems

$$
\begin{gathered}
S_{j}(x)=R_{j}^{-1}\left(x-b_{j}\right), \quad j=1,2, \ldots, m, \quad x \in \mathbb{R}^{d}, \\
S_{j}(x)=R^{-1}\left(x-b_{j}\right), \quad j=1,2, \ldots, m, \quad x \in \mathbb{R}^{d},
\end{gathered}
$$

are called the dual IFSs of (2.1) and (2.2), respectively.

(i) We say that an RIFS satisfies the open set condition (OSC) if its dual IFS does.

(ii) We say that an RIFS satisfies the FTC (see [2]) if its dual IFS does.

REMARK. Since it is required that $\left\{T_{j}\right\}_{j=1}^{m}$ satisfies $\bigcup_{j=1}^{m} T_{j}(M) \subseteq M$ for some uniformly discrete set $M \subseteq \mathbb{R}^{d}$, so not all families of expansive transforms on $\mathbb{R}^{d}$ belong to the family of RIFSs.

Both (2.3) and (2.4) are families of contractive transforms (IFS) defined on $\mathbb{R}^{d}$. Let $K$ be the attractor of these IFSs [2].

It is easy to see the following.

LEMMA 2.4. For the RIFSs (2.1) and (2.2), $P \subset K$.

If $R_{j} \in M_{d}(\mathbb{Z}), b_{j} \in \mathbb{Z}^{d}$ and $M=\mathbb{Z}^{d}$, Strichartz asked the following questions in [4] for the case that $d=1$. 
(1) Does there exist an RIFS of the form (2.1) with a nonoverlapping invariant set but such that the images of $\mathbb{Z}^{d}$ overlap?

(2) Is it possible for such RIFSs to have both overlapping and nonoverlapping invariant sets?

(3) Is it possible for such RIFSs to have an overlapping invariant set but with just a finite number of overlaps?

For (3), we have the following result which answers one aspect of the question.

PROPOSITION 2.5. For the RIFS (2.2) defined on a uniformly discrete set $M \subset \mathbb{R}^{d}$, assume that $a \in P$ is a fixed point of some $T_{j}(1 \leq j \leq m)$ and $F_{a}$ is an infinite set.

(i) The set $F_{a}$ is nonoverlapping if and only if the dual IFS (2.4) satisfies the OSC.

(ii) If $T_{i}\left(F_{a}\right) \cap T_{j}\left(F_{a}\right) \neq \emptyset$, then $T_{i}\left(F_{b}\right) \cap T_{j}\left(F_{b}\right)$ is infinite for any $b \in P$.

Proof. (i) Assume $z \in T_{i}\left(F_{a}\right) \cap T_{j}\left(F_{a}\right)(i \neq j)$, then there exist $I, J$ so that $z=T_{i}\left(T_{I}(a)\right)=T_{j}\left(T_{J}(a)\right)$. Since $a=T_{l}(a)$ for some $T_{l}$, without loss of generality, we assume $|I|=|J|=n$. Note that

$$
T_{i}\left(T_{I}(x)\right)=R^{n+1}(x-a)+T_{j}\left(T_{J}(a)\right), \quad T_{j}\left(T_{J}(x)\right)=R^{n+1}(x-a)+T_{j}\left(T_{J}(a)\right),
$$

we have $T_{i I}=T_{j J}$, so the OSC implies $i I=j J$, a contradiction.

Conversely, assume that $F_{a}$ is nonoverlapping. Let $I=i_{1} i_{2} \ldots i_{n}, J=j_{1} j_{2} \ldots j_{n}$. If $I \neq J$, then $i_{n} i_{n-1} \ldots i_{1} \neq j_{n} j_{n-1} \ldots j_{1}$.

Since $S_{I}=T_{i_{n} i_{n-1} \ldots i_{1}}^{-1}, S_{J}=T_{j_{n} j_{n-1} \ldots j_{1}}^{-1}$ and

$$
\begin{aligned}
& S_{I}(x)=R^{-n}\left(x-b_{i_{n}}-A b_{i_{n-1}}-\cdots-A^{n-1} b_{i_{1}}\right), \\
& S_{J}(x)=R^{-n}\left(x-b_{j_{n}}-A b_{j_{n-1}}-\cdots-A^{n-1} b_{j_{1}}\right) .
\end{aligned}
$$

Note that $F_{a}$ is nonoverlapping, and $T_{i_{n} i_{n-1} \ldots i_{1}}(a)$ and $T_{j_{n} j_{n-1} \ldots j_{1}}(a)$ belong to a uniformly discrete set $M$, hence there exists a constant $c>0$ such that

$$
\begin{gathered}
\left\|x-S_{i_{1} i_{2} \ldots i_{n}}^{-1} \circ S_{j_{1} j_{2} \ldots j_{n}}(x)\right\|=\left\|T_{i_{n} i_{n-1} \ldots i_{1}}(a)-T_{j_{n} j_{n-1} \ldots j_{1}}(a)\right\| \geq c, \\
\forall I \neq J \in \Sigma^{n}, n>0, x \in \mathbb{R}^{d} .
\end{gathered}
$$

Choose a bounded invariant open set $O$ of $\left\{S_{i}\right\}_{i=1}^{m}$, let

$$
N=\sup _{n>0} \sup _{I \in \Sigma^{n}} \#\left\{J \in \Sigma^{n} \mid S_{I}(O) \cap S_{J}(O) \neq \emptyset\right\} .
$$

Since

$$
\begin{aligned}
& S_{I}(O) \cap S_{J}(O) \neq \emptyset \Longleftrightarrow O \cap S_{I}^{-1} S_{J}(O) \neq \emptyset \\
& \quad \Longleftrightarrow O \cap\left(O-T_{i_{n} i_{n-1} \ldots i_{1}}(a)+T_{j_{n} j_{n-1} \ldots j_{1}}(a)\right) \neq \emptyset .
\end{aligned}
$$

Let $B_{\delta}\left(x_{0}\right)$ be a ball contained in $O$ with radius $\delta<c / 2$, then all of the balls of

$$
\begin{aligned}
& \left\{B_{\delta}\left(x_{0}\right)-T_{i_{n} i_{n-1} \ldots i_{1}}(a)+T_{j_{n} j_{n-1} \ldots j_{1}}(a) \mid S_{I}(O) \cap S_{J}(O) \neq \emptyset\right. \\
& \left.\quad J=j_{1} j_{2} \ldots j_{n} \in \Sigma^{n}\right\}
\end{aligned}
$$


are disjoint for any given $n$ and $I=i_{1} i_{2} \ldots i_{n} \in \Sigma^{n}$, and contained in

$$
O_{1}=\left\{x \in \mathbb{R}^{d}|\|x-y\| \leq| O \mid \text { for some } y \in O\right\},
$$

where $|O|$ is the diameter of $O$. Using (2.5) shows that $N<+\infty$. Hence, there is an integer $n_{0}$ and $I \in \Sigma^{n_{0}}$ such that

$$
N=\#\left\{J \in \Sigma^{n_{0}} \mid S_{I}(O) \cap S_{J}(O) \neq \emptyset\right\} .
$$

Fix this $I$, let

$$
V=\bigcup_{n=1}^{+\infty} \bigcup_{J \in \Sigma^{n}} S_{J}\left(S_{I}(O)\right)
$$

It is easy to see that $V$ is a bounded nonempty open set. Furthermore, it is an invariant open set of the dual IFS.

If $S_{i}(V) \cap S_{j}(V) \neq \emptyset$ for some $i \neq j$, then there exist $\tau, \sigma$ such that

$$
S_{i}\left(S_{\tau}\left(S_{I}(O)\right)\right) \cap S_{j}\left(S_{\sigma}\left(S_{I}(O)\right)\right) \neq \emptyset .
$$

Let $|\tau|=n,|\sigma|=k$. Without loss of generality, we can assume $n \geq k$, then $\tau I$ can be written as $\tau I=\tau_{1} \tau_{2}$ with $\left|\tau_{1}\right|=k+n_{0}$. Since $O$ is invariant, so $S_{i}\left(S_{\tau}\left(S_{I}(O)\right)\right)$ $\subseteq S_{i}\left(S_{\tau_{1}}(O)\right)($ see $(2.7))$ implies

$$
S_{i}\left(S_{\tau_{1}}(O)\right) \cap S_{j}\left(S_{\sigma}\left(S_{I}(O)\right)\right) \neq \emptyset .
$$

Hence,

$$
\begin{aligned}
& \left\{J \in \Sigma^{n_{0}+k+1} \mid S_{j}\left(S_{\sigma}\left(S_{I}(O)\right)\right) \cap S_{J}(O) \neq \emptyset\right\} \\
& \quad \supseteq\left\{\left(i \tau_{1}\right)\right\} \cup\left\{(j \sigma J) \mid J \in \Sigma^{n_{0}}, S_{I}(O) \cap S_{J}(O) \neq \emptyset\right\} .
\end{aligned}
$$

Hence, $\#\left\{J \in \Sigma^{n_{0}+k+1} \mid S_{i}\left(S_{\tau}\left(S_{I}(O)\right)\right) \cap S_{J}(O) \neq \emptyset\right\}>N$ by (2.6) and $i \neq j$, it contradicts the definition of $N$.

Therefore, $S_{i}(V) \cap S_{j}(V)=\emptyset$ for all $i \neq j$, that is, the dual IFS (equivalently the RIFS) satisfies the OSC.

(ii) Assume $z \in T_{i}\left(F_{a}\right) \cap T_{j}\left(F_{a}\right), i \neq j$. Note that $a$ is a fixed point of some $T_{l}$, the above proof implies that $T_{i}\left(T_{I}(a)\right)=T_{j}\left(T_{J}(a)\right)$ for some $I=i_{1} i_{2} \ldots i_{p}$, $J=j_{1} j_{2} \ldots j_{p}$. Since $T_{i} T_{I}(x)=R^{p+1}(x-a)+T_{i I}(a), T_{j} T_{J}(x)=R^{p+1}(x-a)$ $+T_{j J}(a)$. Hence, $T_{i I}=T_{j J}$ and so $T_{i}\left(F_{b}\right) \cap T_{j}\left(F_{b}\right) \supseteq T_{i} T_{I}\left(F_{b}\right)$ for all $b \in P$. Since $F_{a}$ is infinite, by Lemma $2.1, F_{b}$ is also infinite, so $T_{i}\left(F_{b}\right) \cap T_{j}\left(F_{b}\right)$ is an infinite set.

The following example answers question (3) in another aspect.

EXAMPLE 2.6. Let $T_{1}(z)=9 z+2, T_{2}(z)=9 z-8, T_{3}(z)=9 z-18$, and $T_{4}(z)$ $=9 z-36$.

Then the dual IFS is

$$
\left\{S_{1}(x)=\frac{1}{9}(x-2), S_{2}(x)=\frac{1}{9}(x+8), S_{3}(x)=\frac{1}{9}(x+18), S_{4}(x)=\frac{1}{9}(x+36)\right\} .
$$


It is easy to see that the dual IFS $\left\{S_{j}\right\}_{j=1}^{4}$ satisfies the OSC with respect to the invariant open interval $V=\left(-\frac{1}{4}, \frac{9}{2}\right)$.

For this RIFS, 1 is the fixed point of $T_{2}$, so $F_{1}$ is nonoverlapping by Proposition 2.5(i).

However, for 0 , the fixed point of $T_{31}$, we have $T_{3}\left(F_{0}\right) \cap T_{4}\left(F_{0}\right)=\{-18\}$ and $T_{i}\left(F_{0}\right)$

$\cap T_{j}\left(F_{0}\right)=\emptyset$ for the other cases of $i \neq j$.

This shows that, if $a \in P$ is not a fixed point of some $T_{i}(i=1,2, \ldots, m), F_{a}$ may be overlapping even if the RIFS satisfies the OSC, that is, the conclusion of Proposition 2.5(i) does not hold without the assumption that $a \in P$ is a fixed point of some $T_{i}(1 \leq i \leq m)$.

PROOF. Let $b_{1}=2, b_{2}=-8, b_{3}=-18$ and $b_{4}=-36$.

Claim 1. If $T_{i_{1} i_{2} \ldots i_{n}}(0)-2=T_{j_{1} j_{2} \ldots j_{p}}(0)$ and $p>0$, then $i_{1}=1, j_{1}=3$ and

$$
T_{i_{2} \ldots i_{n}}(0)=T_{j_{2} \ldots j_{p}}(0)-2
$$

Since $p>0$, so $T_{i_{1} i_{2} \ldots i_{n}}(0)-2=T_{j_{1} j_{2} \ldots j_{p}}(0)$ implies that $n>0$ and

$$
9 T_{i_{2} \ldots i_{n}}(0)+b_{i_{1}}-2=9 T_{j_{2} \ldots j_{p}}(0)+b_{j_{1}} .
$$

Hence, $9 \mid\left(b_{i_{1}}-2-b_{j_{1}}\right)$, this means that $i_{1}=1$ and $j_{1} \in\{3,4\}$. If $j_{1}=4$, then the above equality implies $T_{i_{2} \ldots i_{n}}(0)=T_{j_{2} \ldots j_{p}}(0)-4$, so $9 \mid\left(b_{i_{2}}+4-b_{j_{2}}\right)$, which is impossible. Hence, $j_{1}=3$ and (2.8) hold.

Claim 2. If $T_{i_{1} i_{2} \ldots i_{n}}(0)-2=T_{j_{1} j_{2} \ldots j_{p}}(0)$, then $T_{j_{1} j_{2} \ldots j_{p}}(0)=0$.

Assume $T_{i_{1} i_{2} \ldots i_{n}}(0)-2=T_{j_{1} j_{2} \ldots j_{p}}(0)$ and $p>0$. If $n=1$, Claim 1 implies that $i_{1}=1, j_{1}=3$ and so $T_{j_{2} \ldots j_{p}}(0)=2$, hence $T_{j_{1} j_{2} \ldots j_{p}}(0)=0$. Assume that the conclusion is true for $n<k$. For the case $n=k>1$, Claim 1 implies that $i_{1}=1$, $j_{1}=3$ and

$$
T_{j_{2} \ldots j_{p}}(0)-2=T_{i_{2} \ldots i_{k}}(0) .
$$

Use Claim 1 repeatedly, we have $j_{2}=1, i_{2}=3$ and

$$
T_{i_{3} \ldots i_{n}}(0)-2=T_{j_{3} \ldots j_{p}}(0)
$$

Hence, $T_{j_{3} \ldots j_{p}}(0)=0$ by induction. Therefore, $T_{j_{1} j_{2} \ldots j_{p}}(0)=0$ by $j_{1}=3, j_{2}=1$.

We now turn to prove the conclusion of the example. Assume $z \in T_{i}\left(F_{0}\right) \cap T_{j}\left(F_{0}\right)$. Without loss of generality, we can assume $i>j$. Then there exist $I, J$ such that $T_{i}\left(T_{I}(0)\right)=T_{j}\left(T_{J}(0)\right)$. Hence,

$$
9 T_{I}(0)+b_{i}=9 T_{J}(0)+b_{j}
$$

so $9 \mid\left(b_{i}-b_{j}\right)$. Note that $i>j$, we have $i=4$ and $j=3$. Therefore, $T_{i}\left(F_{0}\right)$ $\cap T_{j}\left(F_{0}\right)=\emptyset$ for other cases of $i \neq j$. 
If $i=4$ and $j=3$, then (2.9) implies

$$
T_{I}(0)-2=T_{J}(0) .
$$

The result of Claim 2 implies $T_{J}(0)=0$, so $T_{3}\left(T_{J}(0)\right)=-18$. Therefore $T_{3}\left(F_{0}\right)$ $\cap T_{4}\left(F_{0}\right)=\{-18\}$ by noting that $T_{3}(0)=T_{41}(0)=-18$. The proof is complete.

For general case, we do not know whether a general conclusion as in the above example is true. We have the following conjecture.

Conjecture. If the RIFS (2.2) satisfies the OSC, then $T_{i}\left(F_{a}\right) \cap T_{j}\left(F_{a}\right)$ is finite for any distinct $i, j$ and $a \in P$.

For questions (1) and (2), we have the following two examples.

EXAmple 2.7. Suppose $T_{1}(z)=4 z, T_{2}(z)=4 z-3, T_{3}(z)=4 z-24$, and $T_{4}(z)=$ $4 z-27$. Then the images of $\mathbb{Z}$ overlap. It is easy to see that $P=\{0,1,8,9\}$ and $F_{0}$, $F_{1}, F_{8}$ and $F_{9}$ are nonoverlapping by Proposition 2.5(i), since the dual IFS

$$
\left\{S_{1}(x)=\frac{1}{4} x, S_{2}(x)=\frac{1}{4}(x+3), S_{3}(x)=\frac{1}{4}(x+24), S_{4}(x)=\frac{1}{4}(x+27)\right\}
$$

generates a tile $[0,3] \cup[6,9]$ and so satisfies the OSC. However, the invariant set $F_{1} \cup F_{9}$ is overlapping, since $T_{13}(9)=48=T_{221}(1)$.

This example shows that all forward orbits of points in $P$ are nonoverlapping does not imply that a union of some of them is nonoverlapping or that the images of $\mathbb{Z}^{d}$ under $T_{j}$ are nonoverlapping. We have answered questions (1) and (2).

Furthermore, we have the following example.

EXAMPLE 2.8. Suppose $T_{1}(z)=4 z$, and $T_{2}(z)=3 z+2$. Then $T_{1}(\mathbb{Z}) \cap T_{2}(\mathbb{Z})$ is infinite. It is easy to see that $P=\{0,-1\}, F_{-1}$ is nonoverlapping, but $T_{1}\left(F_{0}\right) \cap$ $T_{2}\left(F_{0}\right)=\{8\}$.

\section{Dimensions of invariant sets}

We consider the dimensions of invariant sets of the RIFS (2.1) in this section. By Lemma 2.2, we need only consider a forward orbit $F_{a}$ with $a \in P$.

For the RIFS (2.1) and the dual IFS (2.3), let $\gamma_{j}>0$ be the expansive ratio of $T_{j}$. Define

$$
\begin{aligned}
\gamma & =\max _{j}\left\{\gamma_{j}\right\}, \\
\Lambda_{k} & =\left\{I=i_{1} i_{2} \ldots i_{n} \in \Sigma^{n} \mid \gamma_{i_{1} i_{2} \ldots i_{n}}^{-1} \leq \gamma^{-k}<\gamma_{i_{1} i_{2} \ldots i_{n-1}}^{-1}, n>0\right\}, \quad \forall k \in \mathbb{N}, \\
\widetilde{\Lambda}_{k} & =\left\{I=i_{1} i_{2} \ldots i_{n} \in \Sigma^{n} \mid i_{n} \ldots i_{2} i_{1} \in \Lambda_{k}, n>0\right\}, \quad \forall k \in \mathbb{N}, \\
N_{k} & =\#\left\{S_{J} \mid J \in \Lambda_{k}\right\}, \quad \forall k \in \mathbb{N} .
\end{aligned}
$$

We first consider nonoverlapping forward orbits. 
THEOREM 3.1. For an RIFS defined in (2.1), let $F_{a}$ be a forward orbit with $a \in P$. If $F_{a}$ is nonoverlapping, then its dimension is the solution of

$$
\sum_{j=1}^{m} \gamma_{j}^{-\alpha}=1
$$

that is, $\operatorname{dim}_{\mathrm{H}}(K)=\operatorname{dim} F_{a}$.

PROOF. Strichartz has proved the result when $R_{j}=R$ for all $j$ and mentioned the general result in [4]. For completeness, we give a proof here.

Since $F_{a}$ is nonoverlapping, let $\mu$ be the counting measure on $F_{a}$, then

$$
\mu(E)=\sum_{i=1}^{m} \mu \circ T_{j}^{-1}(E), \quad \forall E \subseteq F_{a} .
$$

Consider the dual IFS $\left\{S_{j}(x)=R_{j}^{-1}\left(x-b_{j}\right)\right\}_{j=1}^{m}$, there is a unique nonempty compact set $K$ such that $K=\bigcup_{j=1}^{m} S_{j}(K)$. Since $a \in K$, so $R_{J}^{-1}\left(a-b_{J}\right)=T_{J}^{-1}(a)$ $\in K,\left\|R_{J}^{-1} b_{J}\right\| \leq 2|K|$ for all $J$. Hence, there exists a constant $c>0$ such that $\left\|R_{J}^{-1} b_{J}\right\|<c$ for all $J$, where $R_{J}=R_{j_{1}} R_{j_{2}} \ldots R_{j_{n}}$ and

$$
b_{J}=b_{j_{1}}+R_{j_{1}} b_{j_{2}}+R_{j_{1}} R_{j_{2}} b_{j_{3}}+\cdots+R_{j_{1}} R_{j_{2}} \ldots R_{j_{n-1}} b_{j_{n}} .
$$

Since

$$
T_{J}^{-1}\left(B_{r}\right)=R_{J}^{-1}\left(B_{r}-b_{J}\right)
$$

so

$$
B_{\gamma^{-k-1} r-c} \subseteq T_{J}^{-1}\left(B_{r}\right) \subseteq B_{\gamma^{-k} r+c}, \quad \forall J \in \Lambda_{k} .
$$

By the definition of $\Lambda_{k}$, the identity (3.2) implies

$$
\mu\left(B_{r}\right)=\sum_{J \in \Lambda_{k}} \mu \circ T_{J}^{-1}\left(B_{r}\right)
$$

Use relation (3.3), then

$$
\sum_{J \in \Lambda_{k}} \mu\left(B_{\gamma^{-k-1} r-c}\right) \leq \mu\left(B_{r}\right) \leq \sum_{J \in \Lambda_{k}} \mu\left(B_{\gamma^{-k}+c}\right), \quad \forall k>0 .
$$

Since $\sum_{j=1}^{m} \gamma_{j}^{-\alpha}=1$, by the definition of $\Lambda_{k}$, it is easy to show that $\sum_{J \in \Lambda_{k}}$ $\gamma_{J}^{-\alpha}=1$. Since $\gamma^{k} \leq \gamma_{J}<\gamma^{k+1}$ when $J \in \Lambda_{k}$, we have

$$
\gamma^{k \alpha} \leq \# \Lambda_{k}<\gamma^{(k+1) \alpha} .
$$

For each large $r>0$, let $k=k(r) \in \mathbb{N}$ be such that $\gamma^{k+l} \leq r<\gamma^{k+1+l}$, where $l>0$ is a fixed integer satisfying $\gamma^{l-1}>c$, then (3.4) and (3.5) implies

$$
\alpha \leq \liminf _{r \rightarrow+\infty} \frac{\log \mu\left(B_{r}\right)}{\log r} \leq \limsup _{r \rightarrow+\infty} \frac{\log \mu\left(B_{r}\right)}{\log r} \leq \alpha .
$$


Therefore,

$$
\lim _{r \rightarrow+\infty} \frac{\log \mu\left(B_{r}\right)}{\log r}=\alpha .
$$

We now consider overlapping forward orbits.

LEMMA 3.2. If the dual IFS (2.3) satisfies the FTC, then

$$
\operatorname{dim}_{\mathrm{H}}(K)=\lim _{k \rightarrow+\infty} \frac{\log N_{k}}{\log \gamma^{k}},
$$

where $\operatorname{dim}_{\mathrm{H}}$ is the Hausdorff dimension and $K$ is the attractor of the dual IFS (2.3).

Proof. Note that $N_{k}$ is equal to $\left|\mathcal{V}_{k}\right|$ defined in [2, p. 2]; by [2, Lemma 3.2] and the proof of [2, Theorem 1.1], we see that the limit

$$
\lim _{k \rightarrow+\infty} \frac{\log N_{k}}{\log \gamma^{k}}
$$

exists and

$$
\operatorname{dim}_{\mathrm{H}}(K)=\lim _{k \rightarrow+\infty} \frac{\log N_{k}}{\log \gamma^{k}}
$$

THEOREM 3.3. Let $a \in P$, if the RIFS (2.1) satisfies the FTC and there is a matrix $R$ and integers $k_{j}>0$ such that $R_{j}=R^{k_{j}}$ for all $j$, then

$$
\operatorname{dim} F_{a}=\operatorname{dim}_{\mathrm{H}}(K)=\lim _{k \rightarrow+\infty} \frac{\log N_{k}}{\log \gamma^{k}}
$$

PROOF. Without loss of generality, we assume that there exist $a_{1}, a_{2}, \ldots, a_{d} \in F_{a}$ to be a linearly independent set.

By Lemma 3.2, we only need to show that

$$
\begin{aligned}
\liminf _{r \rightarrow+\infty} \frac{\log N_{k}}{\log \gamma^{k}} & \leq \liminf _{r \rightarrow+\infty} \frac{\log \left(\#\left\{F_{a} \cap B_{r}\right\}\right)}{\log r} \\
& \leq \limsup _{r \rightarrow+\infty} \frac{\log \left(\#\left\{F_{a} \cap B_{r}\right\}\right)}{\log r} \leq \limsup _{k \rightarrow+\infty} \frac{\log N_{k}}{\log \gamma^{k}} .
\end{aligned}
$$

Assume that the dual IFS (2.3) satisfies the FTC with respect to bounded invariant open set $V$. Assume, without loss of generality, $\gamma=\max \left\{\gamma_{j}\right\}=\vartheta^{k_{1}}$, where $\vartheta>1$ is the expansive ratio of the matrix $R$.

Similar to (3.3), there is a constant $c>0$ independent of $k$ such that

$$
T_{J}(a) \in B_{\gamma^{k} c}, \quad \forall J \in \tilde{\Lambda}_{k}
$$

Since $R_{j}=R^{k_{j}}$ and $T_{J}(x)=R_{J}(x-a)+T_{J}(a)$, by the assumption

$$
\gamma=\max \left\{\gamma_{j}\right\}=\vartheta^{k_{1}}, \quad k_{1}=\max _{1 \leq i \leq m}\left\{k_{i}\right\}
$$


If $J \in \widetilde{\Lambda}_{k}$, then $T_{J}(x)=R^{k k_{1}+r}(x)+T_{J}(a)$ for some integral $r$ satisfying $0 \leq r<k_{1}$, so

$$
\#\left\{T_{J} \mid J \in \widetilde{\Lambda}_{k}, T_{J}(a)=z\right\} \leq k_{1}, \quad \forall z \in \mathbb{Z}^{d} .
$$

Therefore,

$$
\#\left(F_{a} \cap B_{\gamma^{k} c}\right) \geq \frac{N_{k}}{k_{1}} .
$$

This means that

$$
\liminf _{k \rightarrow+\infty} \frac{\log N_{k}}{\log \gamma^{k}} \leq \liminf _{r \rightarrow+\infty} \frac{\log \left(\#\left\{F_{a} \cap B_{r}\right\}\right)}{\log r} .
$$

For any fixed $\varepsilon \in(0, \vartheta-1)$, let

$$
M_{\varepsilon}=\varepsilon^{-1} \max \left\{\left\|b_{j}\right\|: 1 \leq j \leq m\right\}
$$

then

$$
\begin{aligned}
\left\|T_{j}(x)\right\| & \geq \gamma_{j}\|x\|-\max \left\{\left\|b_{j}\right\|: 1 \leq j \leq m\right\} \\
& \geq\left(\vartheta^{k_{j}}-\varepsilon\right)\|x\| \geq(\vartheta-\varepsilon)^{k_{j}}\|x\|>\|x\|, \quad \text { if }\|x\|>M_{\varepsilon} .
\end{aligned}
$$

If $J=j_{1} j_{2} \ldots j_{n} \in \Lambda_{k}$, then $\gamma_{j_{1} j_{2} \ldots j_{n}} \geq \gamma^{k}$. Hence,

$$
\left\|T_{J}(x)\right\| \geq(\vartheta-\varepsilon)^{k_{1} k}\|x\|>\|x\|, \quad \text { if }\|x\|>M_{\varepsilon} .
$$

Note that we have assumed that $\gamma=\max \gamma_{j}=\vartheta^{k_{1}}$, that any $z \in F_{a} \cap B_{M_{\varepsilon}(\vartheta-\varepsilon)^{k k_{1}}}$ can be written as $z=T_{J}(b)$ for some $b \in B_{M_{\varepsilon}} \cap F_{a}$ and that $J \in \Sigma^{*}$ with $\gamma_{J}<\gamma^{k+1}$. Hence, there exist $J_{1} \in \bigcup_{j=0}^{\max \left\{k_{i}\right\}} \Sigma^{j}, J_{2} \in \bigcup_{j=0}^{k} \Lambda_{j}$ such that $J=J_{1} J_{2}$, where $\Lambda_{0}=\Sigma^{0}=\emptyset$. Let $Q=\#\left(\bigcup_{j=0}^{\max \left\{k_{i}\right\}} \Sigma^{j}\right), N_{0}=\#\left(B_{M_{\varepsilon}} \cap F_{a}\right)$, then

$$
\#\left(\left\{F_{a} \cap B_{M_{\varepsilon}(\vartheta-\varepsilon)^{k}}\right\}\right) \leq N_{0} Q \sum_{j=1}^{k} N_{j} .
$$

From Lemma 3.2, we see that

$$
\limsup _{k \rightarrow+\infty} \frac{\log \sum_{j=1}^{k} N_{j}}{\log \gamma^{k}}=\limsup _{k \rightarrow+\infty} \frac{\log N_{k}}{\log \gamma^{k}} .
$$

Therefore, (3.8) implies

$$
\begin{aligned}
& \limsup _{r \rightarrow+\infty} \log \left(\#\left\{F_{a} \cap B_{r}\right\}\right) / \log r \\
& \quad=\limsup _{k \rightarrow+\infty} \frac{\log \left(\# \left\{F_{a} \cap B_{\left.\left.M_{\varepsilon}(\vartheta-\varepsilon)^{k k_{1}}\right\}\right)}\right.\right.}{\log \left(M_{\varepsilon}(\vartheta-\varepsilon)^{k k_{1}}\right)} \\
& \quad \leq \frac{\log \vartheta}{\log (\vartheta-\varepsilon)} \limsup _{k \rightarrow+\infty} \frac{\log N_{k}}{\log \gamma^{k}}, \quad \forall \varepsilon \in(0, \vartheta-1) .
\end{aligned}
$$


Let $\varepsilon \rightarrow 0$,

$$
\limsup _{r \rightarrow+\infty} \frac{\log \left(\#\left\{F_{a} \cap B_{r}\right\}\right)}{\log r} \leq \limsup _{k \rightarrow+\infty} \frac{\log N_{k}}{\log \gamma^{k}} .
$$

This relation and (3.7) imply (3.6), and we complete the proof.

COROLlary 3.4. For the RIFS (2.2) and $a \in P$, assume that $R$ is a similar expansive matrix with a similar ratio $\gamma>1$. If the RIFS satisfies the OSC, then the dimension of $F_{a}$ is $\log m / \log \gamma$.

For an RIFS (2.2) satisfying the OSC, a forward orbit may overlap, but this corollary indicates that it will not have too many overlaps.

\section{Acknowledgement}

The author would like to thank Professor K. S. Lau for valuable discussions during the preparation of this paper.

\section{References}

[1] T. Bedford and A. Fisher, 'Analogues of the Lebesgue density theorem for fractal sets of reals and integers', Proc. London Math. Soc. (3) 30 (1992), 95-124.

[2] S.-M. Ngai and Y. Wang, 'Hausdorff dimension of self-similar sets with overlaps', J. London Math. Soc. (2) 63 (2001), 655-672.

[3] N. Nguyen, 'Iterated function systems of finite type and the weak separation property', Proc. Amer. Math. Soc. 130 (2002), 483-487.

[4] R. S. Strichartz, 'Fractal in large', Canad. J. Math. 50 (1996), 638-657.

QI-RONG DENG, Department of Mathematics, Fujiaan Normal University, Fuzhou, 350007, People's Republic of China

e-mail: qrdeng@fjnu.edu.cn 